\title{
RELATIONSHIP BETWEEN AGILITY, LINEAR SPRINTING, AND VERTICAL JUMPING PERFORMANCE IN SLOVENIAN ELITE WOMEN FOOTBALL PLAYERS
}

(1) University School of Physical Education in Wroclaw

original paper

DOI: https://doi.org/10.5114/hm.2020.91281

\section{JOŽEF KRIŽAJ}

Faculty of Sport, University of Ljubljana, Ljubljana, Slovenia

\begin{abstract}
Purpose. The general goal of the study was to investigate the relationship between agility t-test (change of direction speed ability, CODS), 20-m sprint test (S20m) (linear speed ability), and countermovement jump (CMJ) test (lower limb explosive power ability).

Methods. The sample involved 18 elite female football athletes (age: $18.56 \pm 2.24$ years, height: $169.47 \pm 6.48 \mathrm{~cm}$, weight: $63.52 \pm 6.85 \mathrm{~kg}$ ). Pearson correlation coefficients were applied. Multiple linear regression served to explain the relationship between the dependent variable (CODS) and 2 independent variables (S20m and CMJ).

Results. Pearson correlation analysis indicated a moderate relationship between CMJ and CODS performance $(r=-0.59$, $p=0.01)$ and a very strong significant relationship between S20m and CODS performance $(r=0.80, p=0.00)$. The results of multiple linear regression proved that both independent variables (S20m and CMJ) explained about 60.0\% (adjusted $R^{2}$ ) of the total model variance in relation to the dependent variable (CODS), whereas only S20m significantly $(p<0.05)$ explained CODS ability.

Conclusions. The study highlighted the impact of explosive power of lower limbs (CMJ) and linear speed capability (S20m) on agility performance (CODS) in our sample. It suggests that the results of agility, linear sprint, and jumping performance seem to have similar determining factors. It is worthwhile to consider these findings in the training process of female elite football players in order to focus on essential training elements of physical fitness.
\end{abstract}

Key words: $t$-test, power of lower limbs, linear speed ability, female football players

\section{Introduction}

Modern football is rapidly evolving in all components of the game, but especially in terms of physical qualities [1]. The amount of high-speed running during the football match increases constantly at high performance levels [2]. In literature, it is generally considered that sprinting skills in all forms are becoming more and more important in modern football [3].

However, the ability to produce maximal strength levels in lower limbs in the shortest time period is regarded as fundamental to achieve top-level performance in football $[4,5]$. In a soccer game, muscles have to generate and absorb high forces during acceleration and deceleration [6] in linear sprinting actions as well as in agility actions with change of direction. In this context, Nimphius et al. [7] reported a strong relation- ship between relative strength and measures of speed and change of direction ability in female softball athletes. Moreover, Comfort et al. [8] underline the importance of developing high levels of lower limb strength to improve sprint and jump performance in soccer players.

But it appears that different motor skills are needed to perform linear sprinting or sprinting with rapid changes of directions, often specified as agility [9]. Agility in football is thought to be closely linked to explosive power, which represents the above-mentioned ability of a muscle or muscle group to develop maximum force in the shortest possible time [10]. In this context, McFarland et al. [4] found a moderate to strong correlation between sprint speed and explosive power of lower limbs (countermovement jump, CMJ) in female and male football players. Other studies have

Correspondence address: Jožef Križaj, Faculty of Sport, University of Ljubljana, Gortanova 22, 1000 Ljubljana, Slovenia, e-mail: jozef.krizaj@fsp.uni-lj.si

Received: September 18, 2019

Accepted for publication: December 15, 2019

Citation: Križaj J. Relationship between agility, linear sprinting, and vertical jumping performance in Slovenian elite women football players. Hum Mov. 2020;21(2):78-84; doi: https://doi.org/10.5114/hm.2020.91281. 
demonstrated a moderate to strong relationship between explosive power of lower limbs, linear sprint, and agility performance in young soccer players [11]. But also, in other sports, it seems that explosive power of lower limbs has an impact on agility. Barnes et al. [12] argue that the CMJ test (explosive power of lower limbs) is a useful predictor of good agility outcomes in female volleyball players. Ferreira et al. [13] observed that lower limb explosive power appeared a strong predictor of skating linear speed and agility in a sample of young roller hockey elite players.

Despite that, Vescovi and McGuigan [14] found that linear sprinting, agility, and vertical jumping were independent locomotor skills in female athletes, especially with regard to the moderate relationship between $\mathrm{CMJ}$ and linear sprinting results in female high school football players. Little and Williams [15] observed low coefficients of determination $\left(R^{2}\right)$ between agility, acceleration, and maximal speed in a sample of 106 English professional football players. These authors concluded that agility and acceleration as well as maximum speed abilities had different and independent determinants and suggested specific testing and training procedures for each speed-related parameter. It seems that change-of-direction actions are to some extent independent of linear speed actions [16]. Additionally, Sheppard and Young [17] argue that speed and agility express independent physical abilities. They also provide a categorical definition of agility for team sports, describing it as a physical skill that permits athletes to rapidly and efficiently perform a whole-body movement in the form of acceleration or deceleration with a change of direction in response to a stimulus. Therefore, agility in team sports includes not only change of direction abilities but also cognitive elements as anticipation, perception, and decision-making processes [17], and this also in the form of reactive agility $[18,19]$. In more specific terms, first, the athlete needs to receive relevant environmental information in order to process it in relation to previous knowledge and practical values. It is about anticipation and perception of playing situations in the context of cognitive skills [17,20]. Thereupon, the athlete has to decide about the implementation of the appropriate movement [21] to solve the playing situation. In this regard, Gabbett and Abernethy [22] found that high-level athletes had more specific experience of playing situations than low-level athletes; high-level athletes better recognize and anticipate playing situations and exhibit a greater number of correct movement decisions. However, this proves the significance of developing specific playing experiences (knowledge of playing situations, pattern recognition etc.) [17] in order to improve specific information processing [22]. From the practical and theoretical point of view, this proposed modus operandi is similar to the 'principle of specificity' with regard to conditioning issues reported by Reilly et al. [23]. This principle explains that training of physiological determinants has to strain the specific system that the athlete wants to improve for the demands of the sport concerned.

According to Pruna and Bahdur [20], the football field is an unpredictable area with a huge amount of influencing factors, which requires the football player to have different cognitive skills, such as game intelligence, spatial perception, anticipation, reaction time, attention shifting, and pattern recognition. In addition, football is a multidimensional construct, including more than only one stimulus in specific playing situations. It comprises the presence of more stimuli at the same time in terms of movement of team mates, opponents, and the ball. In summary, playing football combines cognitive, physical, and physiological abilities with ball skills.

Here, the dilemma of scientific research in modern football on this specific agility topic begins. What kinds of valid and reliable diagnostic instruments are available in order to maintain useful data for the evaluation of the multidimensional construct of agility in football? There is still not enough scientific evidence for reliable and valid diagnostic measures for change of direction abilities with perception and decision-making processes also with reference to reactive agility $[18,19,24]$.

Therefore, it could be useful, from the diagnostic point of view, to downscale the multidimensional construct of agility to possible determining elements of agility performance respective to basic important categories of locomotor skills (running and jumping) which seem to be measurable, reliable, and valid. In fact, research on agility in team sports, especially in football, offers different points of view. Obviously, there is still not enough evidence that the basic categories of locomotor skills as linear speed performance and explosive power of lower limbs are directly associated with agility abilities [14-16]. There are many studies on the relationship between linear sprinting, change of direction, and explosive power abilities, but the results are discordant. Several results of this research demonstrated strong correlations between these parameters, others pointed at poorer relationships [25]. Differences in test results can have particular causes; perhaps they are based on different diagnostic approaches and research procedures, different sports 
disciplines, and different age categories of selected investigated samples.

However, female and male football players need to have the ability for high speed linear sprinting and for fast change of direction on the pitch. This gives them an advantage over opponents and puts them in a position which could be match-deciding. Especially research on women's football in Slovenia is still lacking a systematic approach; it is generally known that there are differences between women and men regarding locomotor and agility abilities [19]. Until now, limited performance data is available for Slovenian female football players. The development of women's football in Slovenia needs in-depth scientific research that would allow a systematic overview of different abilities of female players and provide coaches with certain feedback on the effects of the training process.

Thus, the general purpose of the study was to investigate the 'downscaled multidimensional construct of agility' among elite women football players. In more specific terms, the main goal was to explore the relationship between the change of direction speed ability (CODS), the linear speed ability (20-m sprint test, $\mathrm{S} 20 \mathrm{~m})$, and lower limb explosive power ability (CMJ). We presumed that there was a relationship between the results of linear speed ability, lower limb explosive power ability (vertical jumping), and the $t$-test describing CODS in elite female football athletes. Furthermore, it was of great interest to explore the effects of linear speed and jumping ability on agility performance measures.

\section{Material and methods}

\section{Subjects}

A total of 18 elite female football players (age: 18.56 \pm 2.24 years, height: $169.47 \pm 6.48 \mathrm{~cm}$, weight: 63.52 $\pm 6.85 \mathrm{~kg}$ ) participated in this study in December 2017, at the end of the first part of Slovenian first women league championship 2017/2018. All female football players were fully informed about the requirements of the research.

\section{Assessment methods}

All assessments took place indoor at the Faculty of Sport, University of Ljubljana, on the same day and under the same conditions. Standardized progressive warm-up program was applied before the evaluation. It included 10 minutes of activation and mobilization exercises, such as light jogging, stretching, skipping drills, and bounding drills, as well as a few acceleration sprints. There was an approximately 10-15-minute rest between the assessments. The trials were implemented by experienced researchers of the faculty.

\section{Lower limb explosive power testing: CMJ}

The lower limb power ability was evaluated with the use of a bilateral force plate (S2P, Ljubljana, Slovenia) by measuring the jump height, which was calculated from the take-off velocity of CMJ with both hands on the waist. The size of each plate was $300 \times$ $600 \mathrm{~mm}$. The signals were acquired at $1000 \mathrm{~Hz}$ and transferred to a personal computer via a USB interface. The ARS software (Analysis and Reporting Software; S2P Ltd., Ljubljana, Slovenia) was used for acquisition and treatment of the selected jump parameters. Best results out of 3 trials were used for further statistical analysis.

\section{Change of direction speed ability: $t$-test}

The assessment of the CODS test was performed by using the Brower Photocell Timing System (Brower, Draper, USA). The athletes were instructed to sprint forward from a standing position to a middle cone $6 \mathrm{~m}$ away and then to perform a side shuffle to the left to a cone $3 \mathrm{~m}$ away from the middle cone. This was followed by a side shuffle to the right with crossing the middle cone to the cone on the right, also placed $3 \mathrm{~m}$ away from the middle cone. After returning by left side shuffles to the middle cone, the test was concluded by running backwards to the starting position. The time was recorded from passing the photocells at the starting position for the first time until the participant crossed the starting position for the second time by running backwards to the starting position as the finish line. All subjects performed 2 trials to the left and right side, with a 5-minute rest for recovery between the trials. The test result was recorded as the best time of the 2 trials to each side. The average time of the best trials to the left and right side was used for further statistical analysis.

\section{Linear speed testing: S20m}

The assessment of the linear sprinting test was also performed with the Brower Photocell Timing System (Brower, Draper, USA). Two photocells were positioned at the start location and at the 20-m distance. The start of the sprint was executed from a standing start position. Once ready, the participants started in 
their own time. The time was recorded from passing the first photocells until the sprinters crossed the 20-m finish line. All subjects performed 2 trials, with a 5-minute rest for recovery between the trials. The fastest time of the S20m test was used for further statistical analysis.

\section{Statistical analysis}

The data were processed by using the IBM SPSS Statistics software for Windows 25.0 (SPSS Inc., Chicago, USA). The analysis included descriptive statistics with means \pm standard deviations, and $95 \%$ confidence intervals. The distribution of each variable was examined for the assumption of normality with the Shapiro-Wilk's normality distribution test. Pearson's product moment-correlation coefficient $(r)$ served to determine correlations. Statistical significance of the results was accepted at $p<0.05$. Multiple linear regression was conducted to explain the relationship between the dependent variable (CODS) and 2 independent variables (S20m and CMJ). The Durbin-Watson (DW) test was applied to examine autocorrelations in the residuals of regression analysis. Furthermore, the analysis of variance (ANOVA) test allowed to determine if the linear regression results were significant.

\section{Ethical approval}

The research related to human use has complied with all the relevant national regulations and institutional policies, and has followed the tenets of the Dec- laration of Helsinki and the ethical guidelines and rules for research with human subjects of the Faculty of Sport, University of Ljubljana.

\section{Informed consent}

Informed consent has been obtained from all individuals included in this study.

\section{Results}

The results of demographic and anthropometric descriptive statistics of the participants (elite female football players) are shown in Table 1.

The results of elite female football players' performance tests are presented in Table 2.

The results of the Pearson product moment-correlation analysis indicated moderate correlation $(r=$ $-0.59, p=0.01$ ) (Table 3) between the explosive power performance of lower limbs (CMJ) and agility performance (CODS). There was a very strong correlation $(r=0.81, p<0.01)$ (Table 3) between S20m and explosive power performance of lower limbs (CMJ). Similarly, the relation between S20m and agility performance (CODS) showed a very strong relationship $(r=0.80, p<0.01)$ (Table 3).

The value of $R^{2}$ explained $65 \%$ of the total variance (Table 4). The adjusted $R^{2}$ coefficient demonstrated that the regression model with 2 predictors still explained $60 \%$ of the total variance. The ANOVA test showed that the independent variables (CMJ and $\mathrm{S} 20 \mathrm{~m}$ ) significantly predicted the dependent variable (CODS)

Table 1. Demographic and anthropometric descriptive statistics of the sample

\begin{tabular}{lrrrrrr}
\hline & & & & & & \multicolumn{2}{c}{$95 \%$ CI } \\
\cline { 3 - 7 } Characteristics & $n$ & Min & Max & Mean $\pm S D$ & LB & UB \\
\hline Age (years) & 18 & 16.30 & 25.00 & $18.56 \pm 2.24$ & 17.44 & 19.67 \\
Weight (kg) & 18 & 49.30 & 76.70 & $63.52 \pm 6.85$ & 61.11 & 66.93 \\
Height (cm) & 18 & 155.70 & 181.00 & $169.47 \pm 6.48$ & 166.25 & 172.69 \\
\hline
\end{tabular}

$S D$ - standard deviation, CI - confidence interval, LB - lower bound, UB - upper bound

Table 2. Descriptive statistics of explosive power, speed, and agility

\begin{tabular}{llccccccc}
\hline \multirow{2}{*}{ Physical ability } & Test & $n$ & Min & Max & Mean $\pm S D$ & \multicolumn{2}{c}{$95 \%$ CI } & Shapiro-Wilk test* \\
\cline { 5 - 7 } & & & & & & LB & UB & 0.30 \\
Explosive power & CMJ (m) & 18 & 0.19 & 0.33 & $0.27 \pm 0.04$ & 0.26 & 0.31 \\
Speed & S20m (s) & 18 & 3.12 & 3.83 & $3.37 \pm 0.19$ & 3.28 & 3.46 & 0.05 \\
Agility & CODS (s) & 18 & 8.15 & 9.86 & $8.92 \pm 0.42$ & 8.70 & 9.13 & 0.73 \\
\hline
\end{tabular}

$S D$ - standard deviation, CI - confidence interval, LB - lower bound, UB - upper bound, CMJ - countermovement jump with arm swing, S20m - 20-m linear sprint, CODS - change of direction speed ability (agility $t$-test to the left and right)

* Shapiro-Wilk's normality distribution test with the significance at $p \geqslant 0.05$ for normal distribution 
J. Križaj, Elite women football players' performance

Table 3. Results of Pearson's correlation analysis $(r)$ in the Slovenian elite women football players

\begin{tabular}{ccccc}
\hline \multicolumn{2}{c}{ Variables } & CODS & S20m & CMJ \\
\hline \multirow{2}{*}{ CODS } & $r$ & 1 & $0.80^{* *}$ & $-0.59^{*}$ \\
& $p$ & - & $<0.01$ & 0.01 \\
\hline \multirow{2}{*}{ S20m } & $r$ & $0.80^{* *}$ & 1 & $-0.81^{* *}$ \\
& $p$ & $<0.01$ & - & $<0.01$ \\
\hline \multirow{2}{*}{ CMJ } & $r$ & $-0.59^{*}$ & $-0.81^{* *}$ & 1 \\
& $p$ & 0.01 & $<0.01$ & - \\
\hline
\end{tabular}

CODS - change of direction speed ability (agility $t$-test to the left and right), S20m - 20-m linear sprint, $\mathrm{CMJ}$ - countermovement jump with arm swing * significant at the level of $p<0.05$,

** significant at the level of $p<0.01$

Table 4. Model summary of multiple regression analysis

\begin{tabular}{lcccc}
\hline$R$ & $R^{2}$ & Adjusted $R^{2}$ & $\begin{array}{c}\text { Standard } \\
\text { error }\end{array}$ & DW \\
\hline 0.80 & 0.65 & 0.60 & 0.27 & 2.12 \\
\hline
\end{tabular}

$R$ - multiple correlation coefficient, $R^{2}$ - coefficient of determination with adjusted $R^{2}$, DW - Durbin-Watson test; independent variables: height of the countermovement jump (CMJ), 20-m linear sprint speed time (S20m); dependent variable: average speed time agility $t$-test to the left and right (CODS)

Table 5. Effects of independent variables on the dependent variable in the linear regression model

\begin{tabular}{ccccccc}
\hline DV & IV & B & $S E$ & $\beta$ & $t$ & $p$ \\
\hline \multirow{2}{*}{ CODS } & S20m & 2.13 & 0.59 & 0.94 & 3.58 & 0.003 \\
& CMJ & 1.79 & 2.64 & 0.18 & 0.68 & 0.508
\end{tabular}

DV - dependent variable, IV - independent variables, B - unstandardized coefficient, $S E$ - standard error,

$\beta$-standardized regression coefficient, $t$ - statistic, $p$ - significance level

$(\mathrm{F}=13.76, p<0.001)$. The DW statistic demonstrated a value nearby $2.0(\mathrm{DW}=2.12)$, which means that there was no autocorrelation detected in the sample.

In the linear regression model (Table 5), only S20m significantly explained the CODS speed ability, while the other parameter, defined as lower limb explosive power ability (CMJ), did not reach the significance level $(p>0.05)$.

\section{Discussion}

The main purpose of the study was to investigate the relationship between the results of the agility $t$-test
(CODS), S20m, and the CMJ test. A very strong correlation was found between the variables of CODS and the S20m test $(r=0.80, p<0.01)$ (Table 3) and between those of CMJ and the S20m test $(r=0.81, p<$ 0.01 ) (Table 3). A moderate correlation was observed between the test results of CMJ and CODS tests $(r=$ $-0.59, p=0.01$ ) (Table 3). It became apparent in our analysis that the aforementioned performance abilities seemed to have similar determining factors in elite Slovenian female football players. The results of this study support those obtained by McFarland et al. [4], who found a moderate to strong correlation between sprint speed and explosive power of lower limbs $(\mathrm{CMJ})$ both in female and in male football players. Moreover, our observations are in line with the research performed by Köklü et al. [11], who proved a similar strong correlation between sprint performance and agility performance $(r=0.74, p=0.01)$ in young male football players. Additionally, the results of this study corroborate other studies $[8,13]$ which underline the importance of developing high levels of lower limb strength to improve sprint and jump performance in soccer players. The initial presumption that there are relationships between the results of linear speed ability, lower limb explosive power ability (vertical jumping), and agility ability ( $t$-test) in elite Slovenian female football athletes can be confirmed. It seems that these variables have similar locomotor determinants. In addition, it was possible to constitute an explanatory multiple regression model (Table 4) which explained 60\% of the model variance (adjusted $R^{2}$ ) in relation to the interaction between the 2 predictors and the dependent variable, which denotes that $40 \%$ of the model variance remained unexplained. Furthermore, we analysed the performance contribution degree of each analysed independent variable of the regression model. In accordance with these statistic results of multiple regression analysis (Table 5), only S20m showed explanatory power for CODS. It was also interesting to note that the lower limb explosive power ability (CMJ) did not help explain the model with regard to the dependent variable (CODS).

However, the evaluated fundamental motor skills specified as explosive power (jumping) and linear sprint ability (running) are in our case related to agility performance in young adult female athletes. Both elements (explosive power and linear sprint ability) could be more or less considered as important factors for successfully playing football. Furthermore, the results of the correlation between CMJ and the S20m test $(r=$ $0.81, p<0.01$ ) (Table 3) illustrate the importance of developing adequate levels of lower limb strength in 
elite female players in order to improve top-level performance in football $[4,5]$.

It could be worthwhile to consider these findings for the training process of female elite football players in order to focus on the fundamental and essential physical training elements, which seems to be important for improving the overall playing capabilities to a higher level of performance. For example, the use of small-sided games in football as a popular training exercise measure should improve different physiological/physical as well as technical/tactical performance components of a football player. But it seems that this popular exercise unit has no significant impact on the lower limb power [26]. On the other hand, Pruna and Bahdur [20] claim that cognitive skills (anticipation, perception, and decision-making processes) of specific football agility cannot be developed through exercise which train just the physical components. The exercises have to address also neural pathways. We completely agree with that statement, but from the practical point of view, it is important to reduce the evaluation contents in modern football to a level which allows coaches to get a diagnostic overview about measurable (reliable and valid) locomotor skills in order to define specific and effective training units, also with regard to injury prevention in the sense of isokinetic knee strength diagnostics [27].

Therefore, we recommend training the abovementioned elementary physical components in terms of explosive power of lower limbs and linear sprint ability as well as change of direction sprints, also separately and stepwise in order to achieve a higher level of performance in these elements.

Thereafter, a process should take place of transmission of these enhanced physical components into complex playing situations with change of direction, perception, and decision-making processes [17] through specific football exercises with the ball. It is about 'learning by playing the game' in all its possible forms with incremental levels of difficulty, for example in small-sided games with changing playing conditions [26]. This simple method includes all cognitive elements $[20,22]$ of successful match performance, such as anticipating and recognizing the playing situation as well as all decision-making processes with the subsequent implementation of adequate movement patterns, including acceleration and deceleration, on higher performance levels with change of direction movements. Such an approach seems to be the most effective training solution for the holistic development of agility in terms of gaining specific playing experiences [17] with the ball, which fit the demands of the sport concerned.
This research work had some limitations that should be considered when interpreting the results of the investigation. The first limitation was the small number of participants, which could have an impact on the test results. Another limitation could occur because the assessments were conducted indoors, on a hard floor, and not in typical environmental conditions for football on natural grass surface. Last but not least, we determined strong correlations between the variables, which showed a relationship but did not confirm the causation. Only the S20m test results seem to have a significant effect on CODS test results.

\section{Conclusions}

In summary, the findings of the present study indicate a significant correlation between explosive power of lower limbs (CMJ), linear speed capability (S20m), and agility performance (CODS) in our specific sample of elite Slovenian female football players. The outcome of this study suggests that the results of agility, linear sprint, and jumping performance seem to have similar determining factors. Furthermore, it appears that these components play a critical role in increasing the playing capabilities of these athletes to a higher level of performance. Coaches should ensure that adult elite female players develop the mentioned physical abilities to a high level of performance as part of a well-planned training program, which, in this case, does not request high equipment costs. The implementation of effective exercises aiming to improve explosive power of lower limbs, speed, and agility appeared to be very useful for reaching a high performance level in female football players.

\section{Disclosure statement}

The author does not have any financial interest and did not receive any financial benefit from this research.

\section{Conflict of interest}

The author states no conflict of interest.

\section{References}

1. Bangsbo J. Physiological demands of football. Sport Sci Exch. 2014;27(125):1-6.

2. Mohr M, Krustrup P, Bangsbo J. Match performance of high-standard soccer players with special reference to development offatigue. J Sports Sci. 2003;21(7):519528; doi: 10.1080/0264041031000071182.

3. Haugen T, Tønnessen E, Hisdal J, Seiler S. The role and development of sprinting speed in soccer. Int J Sports Physiol Perform. 2014;9(3):432-441; doi: 10.1123/ ijspp.2013-0121. 
4. McFarland IT, Dawes JJ, Elder CL, Lockie RG. Relationship of two vertical jumping tests to sprint and change of direction speed among male and female collegiate soccer players. Sports. 2016;4(11):1-7; doi: 10.3390/ sports4010011.

5. Wisløff U, Castagna C, Helgerud J, Jones R, Hoff J. Strong correlation of maximal squat strength with sprint performance and vertical jump height in elite soccer players. Br J Sports Med. 2004;38(3):285-288; doi: 10.1136/bjsm.2002.002071.

6. Maly T, Zahalka F, Mala L. Isokinetic strength performance profile of young national soccer players. Acta Kinesiol. 2015;9(Suppl. 1):61-64.

7. Nimphius S, McGuigan MR, Newton RU. Relationship between strength, power, speed, and change of direction performance of female softball players. J Strength Cond Res. 2010;24(4):885-895; doi: 10.1519/ JSC.0b013e3181d4d41d.

8. Comfort P, Stewart A, Bloom L, Clarkson B. Relationships between strength, sprint, and jump performance in well-trained youth soccer players. J Strength Cond Res. 2014;28(1):173-177; doi: 10.1519/JSC.0b013e31829 $1 \mathrm{~b} 8 \mathrm{c} 7$.

9. Mujika I, Santisteban J, Castagna C. In-season effect of short-term sprint and power training programs on elite junior soccer players. J Strength Cond Res. 2009; 23(9):2581-2587; doi:10.1519/JSC.0b013e3181bc1aac.

10. Thomas K, French D, Hayes PR. The effect of two plyometric training techniques on muscular power and agility in youth soccer players. J Strength Cond Res. 2009;23(1):332-335; doi: 10.1519/JSC.0b013e318183 a01a.

11. Köklü Y, Alemdaroğlu U, Özkan A, Koz M, Ersöz G. The relationship between sprint ability, agility and vertical jump performance in young soccer players. Sci Sports. 2015;30(1):e1-e5; doi: 10.1016/j.scispo.2013.04.006.

12. Barnes JL, Schilling BK, Falvo MJ, Weiss LW, Creasy AK, Fry AC. Relationship of jumping and agility performance in female volleyball athletes. J Strength Cond Res. 2007;21(4):1192-1196; doi: 10.1519/R-22416.1.

13. Ferreira A, Enes C, Leao C, Goncalves L, Clemente FM, Lima R, et al. Relationship between power condition, agility, and speed performance among young roller hockey elite players. Hum Mov. 2019;20(1):24-30; doi: 10.5114/hm.2019.79040.

14. Vescovi JD, McGuigan MR. Relationships between sprinting, agility, and jump ability in female athletes. J Sports Sci. 2008;26(1):97-107; doi: 10.1080/0264 0410701348644.

15. Little T, Williams AG. Specificity of acceleration, maximum speed, and agility in professional soccer players. J Strength Cond Res. 2005;19(1):76-78; doi: 10.1519/ 14253.1.

16. Young W, Benton D, Duthie G, Pryor J. Resistance training for short sprints and maximum-speed sprints. Strength CondJ.2001;23(2):7-13; doi: 10.1519/00126548200104000-00001.
17. Sheppard JM, Young WB. Agility literature review: classifications, training and testing. J Sports Sci. 2006; 24(9):919-932; doi: 10.1080/02640410500457109.

18. Čoh M, Vodičar J, Žvan M, Šimenko J, Stodolka J, Rauter $S$, et al. Are change-of-direction speed and reactive agility independent skills even when using the same movement pattern? J Strength Cond Res. 2018;32(7): 1929-1936; doi: 10.1519/JSC.0000000000002553.

19. Rauter S, Čoh M, Vodičar J, Žvan M, Križaj J, Šimenko J, et al. Analysis of reactive agility and change-of-direction speed between soccer players and physical education students. Hum Mov. 2018;19(2):68-74; doi: 10.5114/hm.2018.74061.

20. Pruna R, Bahdur K. Cognition in football. J Nov Physiother. 2016;6:316; doi: 10.4172/2165-7025.1000316.

21. Broadbent DP, Causer J, Williams AM, Ford PR. Perceptual-cognitive skill training and its transfer to expert performance in the field: future research directions. Eur J Sport Sci. 2015;15(4):322-331; doi: 10.1080/ 17461391.2014 .957727$.

22. Gabbett TJ, Abernethy B. Expert-novice differences in the anticipatory skill of rugby league players. Sport Exerc Perform Psychol. 2013;2(2):138-155; doi: 10.1037/a0031221.

23. Reilly T, Morris T, Whyte G. The specificity of training prescription and physiological assessment: a review. J Sports Sci. 2009;27(6):575-589; doi: 10.1080/02640 410902729741.

24. Oliver JL, Meyers RW. Reliability and generality of measures of acceleration, planned agility, and reactive agility. Int J Sports Physiol Perform. 2009;4(3):345354; doi: 10.1123/ijspp.4.3.345.

25. Popowczak M, Rokita A, Świerzko K, Szczepan S, Michalski R, Maćkała K. Are linear speed and jumping ability determinants of change of direction movements in young male soccer players? J Sport Sci Med. 2019;18(1):109-117.

26. Clemente FM, Nikolaidis PT, Van der Linden CMI, Silva B. Effects of small-sided soccer games on internal and external load and lower limb power: a pilot study in collegiate players. Hum Mov. 2017;18(1):50-57; doi: 10.1515/humo-2017-0007.

27. Kabaciński J, Murawa M, Fryzowicz A, Dworak LB. A comparison of isokinetic knee strength and power output ratios between female basketball and volleyball players. Hum Mov. 2017;18(3):40-45; doi: 10.1515/ humo-2017-0022. 